\title{
Expanding clinical phage microbiology: simulating phage inhalation for respiratory tract infections
}

\author{
Shira Ben Porat ${ }^{1,2,7}$, Daniel Gelman ${ }^{1,2,3,7}$, Ortal Yerushalmy $\mathbb{C}^{1}$, Sivan Alkalay-Oren ${ }^{1}$, \\ Shunit Coppenhagen-Glazer ${ }^{1}$, Malena Cohen-Cymberknoh ${ }^{4,5}$, Eitan Kerem ${ }^{4,5}$, Israel Amirav $\mathbb{C}^{6}$, \\ Ran Nir-Paz ${ }^{3,5,8}$ and Ronen Hazan $\mathbb{1}^{1,8}$
}

${ }^{1}$ Institute of Biomedical and Oral Research, Faculty of Dental Medicine, The Hebrew University of Jerusalem, Jerusalem, Israel. ${ }^{2}$ Dept of Military Medicine, Faculty of Medicine, The Hebrew University of Jerusalem, Jerusalem, Israel. ${ }^{3}$ Dept of Clinical Microbiology and Infectious Diseases, Hadassah-Hebrew University Medical Center, Jerusalem, Israel. ${ }^{4}$ Pediatric Pulmonology Unit and Cystic Fibrosis Center, Hadassah Medical Center, Jerusalem, Israel. ${ }^{5}$ Faculty of Medicine, Hebrew University of Jerusalem, Jerusalem, Israel. ${ }^{6}$ Pediatric Pulmonary Unit, Dana-Dwek Children's Hospital, Tel Aviv, Israel. ${ }^{7}$ These authors contributed equally. ${ }^{8}$ These authors contributed equally.

Corresponding author: Ronen Hazan (ronenh@ekmd.huji.ac.il)

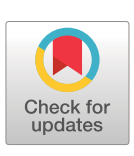

This version is distributed under the terms of the Creative Commons Attribution NonCommercial Licence 4.0. For commercial reproduction rights and permissions contact permissions@ersnet.org

Received: 30 May 2021 Accepted: 5 Aug 2021

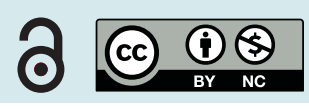

Shareable abstract (@ERSpublications)

Phage therapy can be used against infectious diseases if personally tailored. Using a 3D airway model, this study shows that phage delivery to the respiratory tract by inhalation is unpredictable and requires precise evaluation. https://bit.ly/3ATdzr5

Cite this article as: Ben Porat S, Gelman D, Yerushalmy O, et al. Expanding clinical phage microbiology: simulating phage inhalation for respiratory tract infections. ERJ Open Res 2021; 7: 003672021 [DOI: 10.1183/23120541.00367-2021].

\section{Abstract}

Phage therapy is a promising antibacterial strategy for resistant respiratory tract infections. Phage inhalation may serve this goal; however, it requires a careful assessment of their delivery by this approach. Here we present an in vitro model to evaluate phage inhalation. Eight phages, most of which target pathogens common in cystic fibrosis, were aerosolised by jet nebuliser and administered to a real-scale computed tomography-derived 3D airways model with a breathing simulator. Viable phage loads reaching the output of the nebuliser and the tracheal level of the model were determined and compared to the loaded amount. Phage inhalation resulted in a diverse range of titre reduction, primarily associated with the nebulisation process. No correlation was found between phage delivery to the phage physical or genomic dimensions. These findings highlight the need for tailored simulations of phage delivery, ideally by a patient-specific model in addition to proper phage matching, to increase the potential of phage therapy success.

\section{Introduction}

Phage therapy refers to the use of bacteriophages (phages), bacterial viruses, as antimicrobial agents. Lytic phages can propagate in the presence of their bacterial hosts while sequentially lysing proximal bacterial cells. This ability enables them to penetrate and destroy biofilm and co-evolve with bacterial targets [1] The emerging threat of antimicrobial resistance has re-introduced this previously neglected method and its advantages to the clinical practice, with an increasing number of reports applying phages for infectious diseases in recent years [2-4].

One significant potential target of phage therapy is in the treatment of life-threatening pulmonary infections [5]. These may include acute conditions such as multidrug-resistant (MDR) pneumonia among ventilated patients $[6,7]$, or chronic conditions such as cystic fibrosis (CF), an inherited life-shortening disease associated with recurrent lung infections [8-10]. Phages have been recently used in several clinical cases of pulmonary infections, such as the use of intravenous (i.v.) phages in a 26-year-old CF patient with MDR Pseudomonas aeruginosa pneumonia [9], a case of a 12-year-old lung-transplanted CF patient treated by inhaled phages and by direct phage administration via therapeutic bronchoscopy for Achromobacter xylosoxidans [10], and the treatment of six individuals with CF or non-CF bronchiectasis, treated by nebulised phages for $P$. aeruginosa [11]. Evidently, these published cases differ in treatment 
protocols and specifically in the method for phage delivery, ranging between systemic administration and direct inhalation.

The current understanding of the importance of personalised and accurate phage matching for therapy dictates the use of simulations and in vitro predictions during phage therapy treatment design [12]. Phage inhalation is not exceptional in this manner, as differences in phages' biochemical and physical characteristics may cause suboptimal delivery to the target site, as may be seen with inhaled antibiotics if using inappropriate agents or delivery devices [13]. Thus, the efficacy of phage inhalation should be tested in advance.

Several in vitro studies have previously assessed phage inhalation by comparing phage recovery at the output of various nebulisers $[14,15]$. However, phage concentration reaching the lower respiratory tract was usually not measured but only estimated, for instance, according to the aerodynamic diameter of the released particles [14].

To bridge the gap, we applied a real-scale 3D model of a patient's CT-derived airways. This model, previously used for in vitro aerosol studies [16, 17], was adapted here to evaluate phage delivery to the lungs by inhalation.

Methods

Phages and bacteria

Phages targeting the following seven bacterial strains, most of which are associated with CF, were used (table 1): P. aeruginosa strains PA14 and PAR1, Mycobacterium abscessus MAC107, Burkholderia cepacia strains BCC129 and BCC378, Staphylococcus aureus SAR1 and Staphylococcus epidermidis SE52. Except for the laboratory strain P. aeruginosa PA14, all isolates were obtained from the Clinical Microbiology Department at the Hadassah Medical Center, Israel. S. epidermidis was grown in brain heart infusion (BHI) broth (Difco, Sparks, MD, USA), and all other bacteria were grown in Luria-Bertani (LB) broth (Difco), all at $37^{\circ} \mathrm{C}$ under aerobic conditions shaken on a rotary shaker at $200 \mathrm{xg}$. BHI or LB agar (1.5\%) plates were used for isolation streaks and phage enumeration, respectively. All phages were isolated as part of the Israeli Phage Bank [18]. The phages were propagated on their respective target bacterial hosts as previously described [19].

\section{Phage enumeration}

Phage titres were determined by the double-layered agarose assay as previously described [20]. Briefly, agar plates were covered with pre-warmed agarose $(0.5 \%)$, to which $300 \mu \mathrm{L}$ of the relevant overnight bacterial culture was added. The phage lysates were serially diluted by 10 -fold, and $5-\mu \mathrm{L}$ drops of these dilutions were spotted on the double-layered plates, which were then incubated overnight at $37{ }^{\circ} \mathrm{C}$. The number of plaques on the plates was then counted to calculate the initial phage titres, presented as plaque-forming units (PFU) $\cdot \mathrm{mL}^{-1}$.

\section{Phage recovery from filters}

Phages were recovered from filters placed across the model to evaluate phage viability at these sites. Following each run of the nebuliser, the used filter was left overnight in a sterile tube containing $15 \mathrm{~mL}$ of fresh media at $4^{\circ} \mathrm{C}$, followed by phage enumeration.

To estimate the fraction of phage titre reduction caused by the used filters ( $F r$ - filter reduction fraction), $0.2 \mathrm{~mL}$ of each phage in a known concentration was dropped directly on the pads. The filters were then

\section{TABLE 1 Characteristics of the phages used in the study}

\begin{tabular}{lccccc} 
Phage & \multicolumn{1}{c}{ Host } & Taxonomy & Genome size bp & Genome structure & GenBank accession \\
\hline BCSR5 & Burkholderia cepacia BCC378 & Myoviridae & 227351 & Circular & MW460245.1 \\
SAOMS1 & Staphylococcus aureus SAR1 & Herelleviridae & 140135 & Circular & MW460250.1 \\
BCSR129 & B. cepacia BCC197 & Myoviridae & 66147 & Circular & MW460247.1 \\
\hline PASA16 & Pseudomonas aeruginosa PA14 & Myoviridae & 66127 & Circular & MT933737.1c \\
Itty13 & P. aeruginosa PAR1 & Podoviridae & 61818 & Linear & MW460249.1 \\
Mac02 & Mycobacterium abscessus c107 & Siphoviridae & 48239 & Circular & MW460248.1 \\
Mac07 & M. abscessusx c107 & Siphoviridae & 47836 & Linear & MZ152914 \\
SeAlphi & Staphylococcus epidermidis SE52 & Podoviridae & 18368 & Circular & MZ152915 \\
\hline
\end{tabular}


left to dry and later treated as described above. For each phage, the Fr was calculated as the proportion between viable phages retrieved from the filters to their original inserted titre (table 2).

\section{Phage nebulisation}

The commonly clinically used jet nebuliser Pari LC Sprint (Pari GmbH, Starnberg, Germany), combined with Pari TurboBoy SX Compressor (Pari GmbH), was used for phage nebulisation (figure 1). In each run, $3 \mathrm{~mL}$ of phage lysate in a previously determined concentration was pipetted into the inhalation device reservoir, and the compressor was operated for $15 \mathrm{~min}$. For evaluation of phage viability following nebulisation, a Pari filter/valve set (Pari GmbH) filled with single-use filter pads (Pari GmbH) was connected to the output of the nebuliser to collect aerosol particles (figure 1a).

\section{D face and airways model construction}

The ability of nebulised phages to reach the lower respiratory tract (lung delivery) was evaluated using a 3D reconstruction model of a face and respiratory tract airways (figure $1 \mathrm{~b}$ ). This technique is commonly used in in vitro aerosol delivery studies to quantify aerosol lung delivery [16, 17, 21]. For the face and airways model creation, CT (Brilliance CT, 64-channel scanner; Philips Healthcare, Best, The Netherlands) scans of 5-year-old children with no craniofacial anomalies were obtained for medical indications. The data were digitised and converted to electronic files. Technical acquisition parameters included: $64 \times 0.625$ collimation, 0.75 -s pitch, $120 \mathrm{kV}, 100 \mathrm{~mA}$ and a 2-s rotation time. The thickness of a slice was $2.5 \mathrm{~mm}$, with increments of $1.25 \mathrm{~mm}$. Slices were stored in Digital Imaging and Communications in Medicine (DICOM) format. Subsequently, the slices were joined together to compose a 3-dimensional reconstruction image, which was stored in Standard Transformation Language format, and later transferred over local area network for further analysis. The scans were reconstructed and stored in stereolithography format from which the model was then printed using rapid prototype development techniques. We constructed the model using a photopolymer resin (PolyJet FullCure 720; Objet Geometries Ltd., Sint-Stevens-Woluwe, Belgium), a transparent, stiff material widely used in rapid product development techniques on an Objet Eden 330 3D Printer (Objet Geometries, Rehovot, Israel). Printing layer thickness was $0.016 \mathrm{~mm}$. Anatomical structures included were all air conducting parts from the nostrils to $5 \mathrm{~mm}$ below the glottis.

In addition, a breathing simulator (Harvard Apparatus Respirator Model 665; Harvard Apparatus, Holliston, MA, USA) was connected through the distal orifice of the model. The breathing simulator generated a standard waveform at a preset frequency $\left(\mathrm{f}=20\right.$ breaths $\left.\cdot \mathrm{min}^{-1}\right)$ and tidal volume $(\mathrm{Vt}=80 \mathrm{~mL})$, representing breathing patterns appropriate for the model size. A flow meter (TSI Model 4043; TSI Inc., Shoreview, MN, USA) was connected to the breathing simulator and to the filter/valve set to ensure the flow characteristics through the model (figure 1c).

\section{Lung delivery of phages}

The face and airways model was connected to the Pari LC Sprint nebuliser (Pari GmbH) by a tightly connected face mask (Pari $\mathrm{GmbH}$ ) at the output of the nebuliser (figure 1c). In each run, $3 \mathrm{~mL}$ of phage lysate was pipetted into the nebuliser reservoir, and the compressor was operated for $15 \mathrm{~min}$. For evaluation of phage viability at the tracheal level, the Pari filter/valve set (Pari GmbH) was now placed at the distal portion of the airways model to collect aerosol particles reaching this site (figure 1c).

\section{Phage physical characteristics determination}

Transmission electron microscopy (TEM) was used for measurement of phage particle size. Phage samples were prepared as previously described [19]. Briefly, $1 \mathrm{~mL}$ of phage lysate containing at least $10^{8}$ $\mathrm{PFU} \cdot \mathrm{mL}^{-1}$ was centrifuged at $12225 \mathrm{~g}$ (WiseSpin ${ }^{\circledR}$ CF-10; Daihan Scientific, Gangwon-do, South Korea)

\section{TABLE 2 Filter reduction fraction (Fr) of each phage}

\begin{tabular}{lc} 
Phage & $F r$ \\
\hline BCSR5 & 0.178 \\
SAOMS1 & 0.222 \\
BCSR129 & 0.132 \\
PASA16 & 0.085 \\
Itty13 & 0.053 \\
Maco2 & 0.036 \\
Maco7 & 0.015 \\
SeAlphi & 0.58 \\
\hline
\end{tabular}


a)

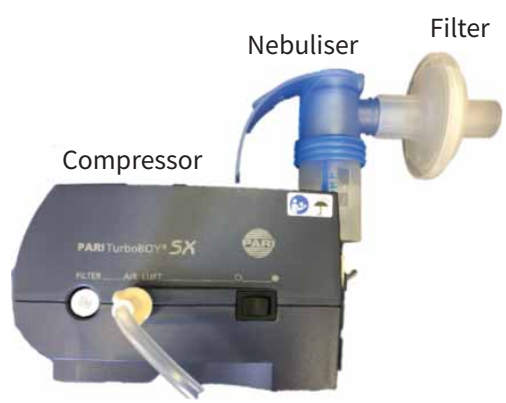

b)

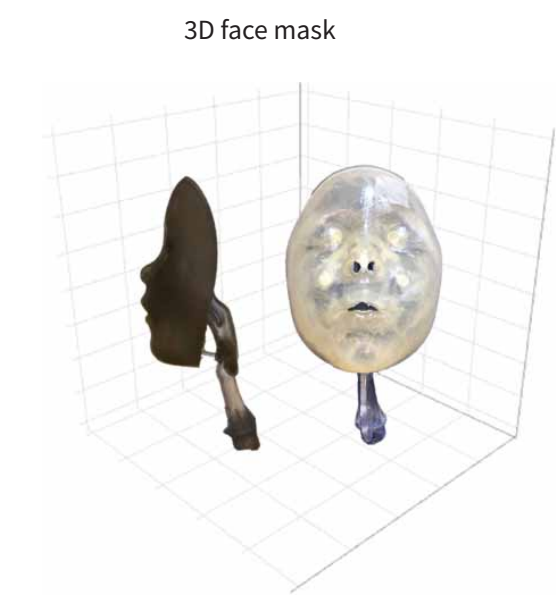

c)

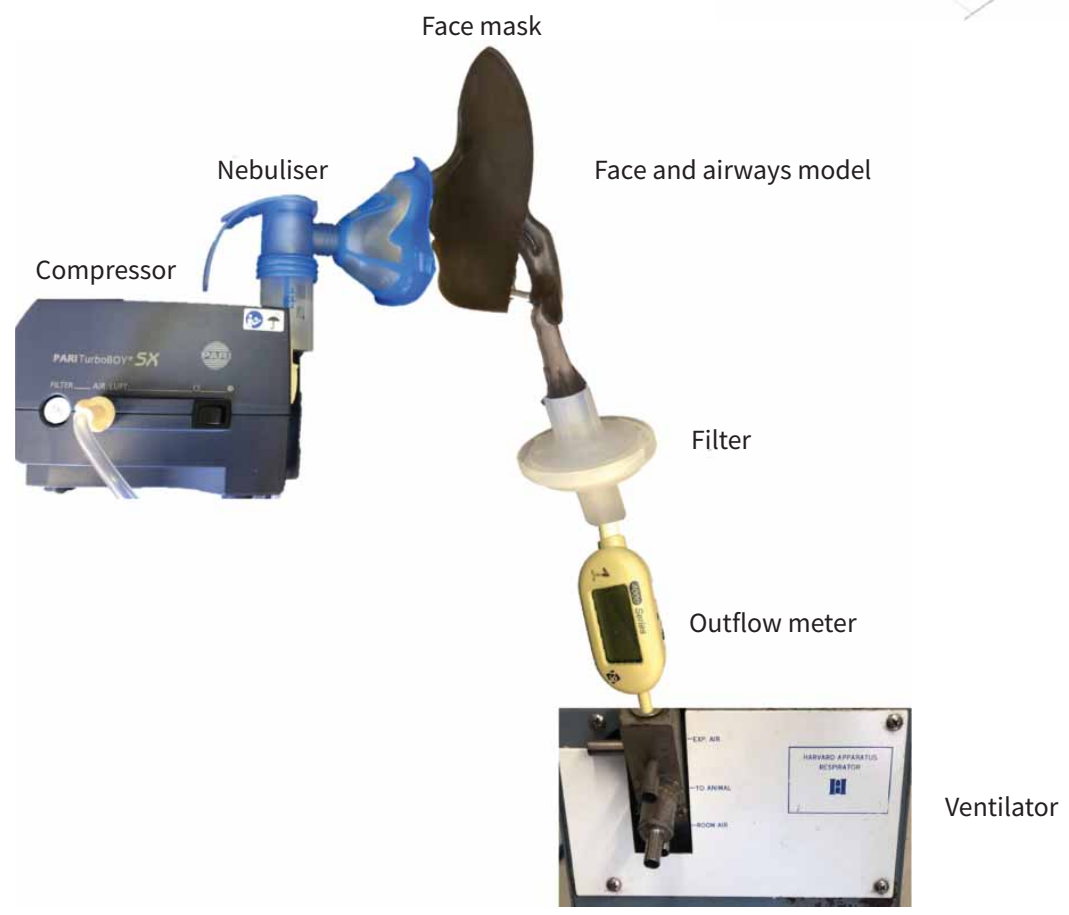

FIGURE 1 Model for evaluation of phage nebulisation and delivery. a) Demonstration and schematic representation of phage nebulisation. Filter pads were placed at the output of the nebuliser to allow phage capture after each run of nebulisation. b) Anterior and lateral views of the 3D face and respiratory airways model. c) Demonstration and schematic representation of phage inhalation. Phages were nebulised and delivered by a face mask to the face and respiratory tract model, connected to a breathing simulator. Phages were captured by filter pads placed at the distal orifice of the airways model.

for $1.5 \mathrm{~h}$ at room temperature. The supernatant was discarded, and the pellet was resuspended in $200 \mu \mathrm{L}$ of $5 \mathrm{mM} \mathrm{MgSO}_{4}$ and left for $24 \mathrm{~h}$ at $4^{\circ} \mathrm{C}$. For grid preparation, $10 \mu \mathrm{L}$ of the phage mixtures was added to $30 \mu \mathrm{L}$ of $5 \mathrm{mM} \mathrm{MgSO}_{4}$, and the grids were placed on the drops with the carbon side facing down. After a minute, the grid was placed on a $30-\mu \mathrm{L}$ drop of NanoVan (Nanoprobes, Yaphank, NY, USA), followed by an additional incubation for 5-10 s. A transmission electron microscope (Jeol, TEM 1400 plus) with a charge-coupled device camera (Gatan Orius 600) was used to capture images. Phage particle size was determined as the mean of measurements capturing 4-14 (median 8.5) virions for each phage, using ImageJ 1.53 h software (http://imagej.nih.gov/ij/).

\section{Statistical analysis}

Phage titres were compared by an unpaired t-test. A significance level of 0.05 was used to determine the statistical difference of viable phage loads due to nebulisation or lung delivery. Pearson's correlation test 
was used for correlation plots. GraphPad prism 8.02 Build 263 (GraphPad Software, Inc, San Diego, CA, USA) was used for statistical tests. Adobe Illustrator (CC release 21.0.2) was used for graph drawing.

Results

The effect of inhalation on viable phage titres

We evaluated the delivery of eight different phages to the lungs using an anatomically accurate face and airways model with corresponding simulation of breathing patterns. The tested phages were selected due to their ability to target pathogens isolated from CF patients (table 1). An additional phage targeting $S$. epidermidis was tested due to a remarkable difference in morphology.

The phages were nebulised and administered to the model. After phage titre correction according to the $\mathrm{Fr}$ (table 2), we found a diverse range of titre drops during phage inhalation to the lungs between the various phages (figure 2). This reduction ranged from 2.8-log for the phage Maco7 to only 0.2- log reduction with the phage SeAlphi. Overall, seven of the eight tested phages presented a significant titre drop at the tracheal level, simulating their deposition in the lower respiratory tract (figure 2).

\section{Determination of phage titre drop during the process of nebulisation}

In order to better understand the factors associated with phage titre reduction during inhalation, we evaluated phage viability following the nebulisation process alone. Viable aerosolised phages were now captured at the output of the nebuliser, representing their titre at the oropharyngeal level. We found that a significant reduction in viable phage loads was also observed following nebulisation for all phages presenting decreased titres at the tracheal level (figure 2). The nebulisation process also led to a diverse range of phage titre reductions, between 2.45-log for the phage BCSR129 to only 0.15- log for the phage SeAlphi. Furthermore, in all phages presenting a significant titre drop at the tracheal level, no significant difference was found between their loads at the trachea and at the output of the nebuliser. This demonstrates that this reduction is highly due to the nebulisation process itself.

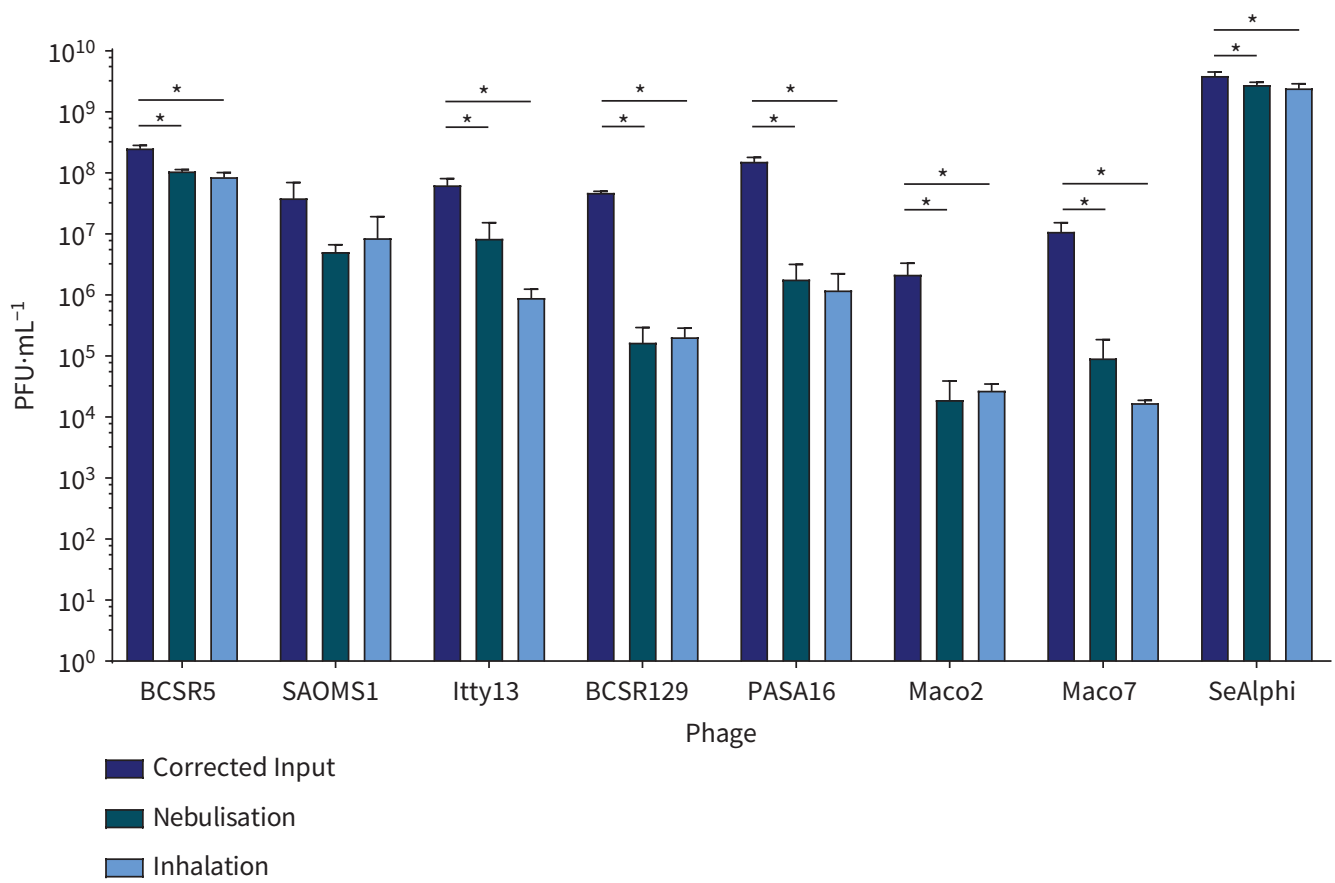

FIGURE 2 Titre reduction of phages following nebulisation and inhalation to the airways model. For each phage, the initial phage titre inserted to the nebuliser, corrected according to the filter reduction fraction (Corrected Input), is compared to the phage titres retrieved from filters placed at the output of the nebuliser (Nebulisation) and at the tracheal level of the respiratory tract model (Inhalation). Three separate runs were made for each phage in every condition. Asterisks represent significant difference $(p<0.05)$. PFU: plaque-forming units. 
Effect of phage characteristics on titre drop during inhalation

Next, we turned to test for intrinsic phage characteristics which may affect their delivery. That way, if a correlation between specific phage characteristics and their ability to pass the model is found, it will allow to better predict the desired phages to administer and their required dosage. The tested traits included the size of the capsids, tail length, total phage length and genome size. However, no correlation was found between these tested characteristics and the phage deposition at the tracheal level (figure 3).

\section{Discussion}

In this study we have simulated phage inhalation using a dedicated real size 3D reconstruction model of human airways, with an adjusted breathing simulator. To the best of our knowledge, this is a first of a kind simulation allowing to directly measure phage delivery to the lungs in realistic human aerodynamic characteristics. Using this model, we found that there is a high variation in the levels of phage titre reduction following inhalation among different phages. Moreover, this reduction was mostly attributed to the nebulisation process itself and was significant in seven of eight tested phages.

Previous reports have evaluated the ability of phages to be aerosolised, usually by direct measurements of their titres at the output of the nebulisation device. SАнота et al. [14] described the recovery of specific anti-pseudomonal phages following nebulisation. In their study, the total number of viable phages leaving a jet nebuliser ranged between $15 \%$ for one phage to $2 \%$ for another respectively to their original amount. CARRIGY et al. [15] have shown that the same anti-tuberculosis phage presented highly diverse levels of titre reductions, ranging between 0.4- and 3.7-logs, when nebulised by different devices such as a vibrating-mesh nebuliser, a jet nebuliser and a soft mist inhaler. In another study, AstudiLlo et al. [22] have supplied visual proof for the structural changes of phages upon nebulisation by different methodologies. Using TEM, they visualised that nebulisation of a specific anti-pseudomonal phage by jet, vibrating-mesh or static-mesh nebulisers resulted in high levels of phage tail separation. As in the current study, these reports emphasise that phage titre may drop during nebulisation, and that the most appropriate phages chosen for inhalation, as well as the nebulisation methodology itself, may vary.

LEUNG et al. [23] have suggested that nebulisation-induced titre loss was particularly correlated with the tail length of the phage. In their study, viable phage titres dropped by 0.04- to 2-logs following jet nebulisation, in correlation with phage morphology. However, in our study, using different phages with a wide range of different characteristics, including short-tailed Podoviridae as well as Myoviridae and Siphoviridae, no correlation to tail length was observed. Furthermore, we have also found no correlation between phage inhalation capabilities to phage size, genome size, taxonomy or to the bacterial host. Thus, we conclude that routine genomic and morphological characterisation of the phages may not be sufficient to predict their nebulisation and inhalation abilities. Accordingly, specific in vitro evaluations should be performed to address phage delivery prior to inhalation treatment. This point was recently demonstrated by GuILLON et al., who measured phage delivery by nebulisation in vitro, to select the most appropriate inhalation interface and phage dosage before administering aerosolised phages to pigs suffering from pneumonia [6].

The rationale behind phage inhalation is similar to the concept of nebulised antibiotics, which may potentially reach high intrapulmonary concentrations and improve outcomes of pulmonary infections [13]. Nebulised antibiotics were mainly studied among ventilated patients with pneumonia, and recent large trials in the field have no benefit from this technique [24, 25]. Nevertheless, phage inhalation represents a novel therapeutic approach due to significant differences in the antibacterial effects of these agents and the differences in the potential treatment population. For instance, the ability of phages to multiply in the presence of the bacterial hosts and to destroy biofilm and their ability to synergise with other antibiotics [1] may lead to a better antibacterial effect in the lung tissue, thus leading to improved treatment outcomes. However, as seen in the current study, only accurate in vitro simulations of phage delivery and their antibacterial efficacy in specific clinical settings should dictate treatment design.

The current study has several limitations. Firstly, although the presented model supplies accurate anatomical characteristics, it does not include the complete physiological conditions found in the respiratory tract. These include the complex tissue environment, the mucociliary structures and the immune system effects. Thus, an additional reduction in phage titres may be expected in the clinical setting, requiring additional studies using animal models [6, 26]. Secondly, no particle size was measured in this study. Given the importance of targeting phage delivery to the deep lung alveoli, it would be helpful in future research to address this issue and specifically use a model of the lung tissue. 
a)

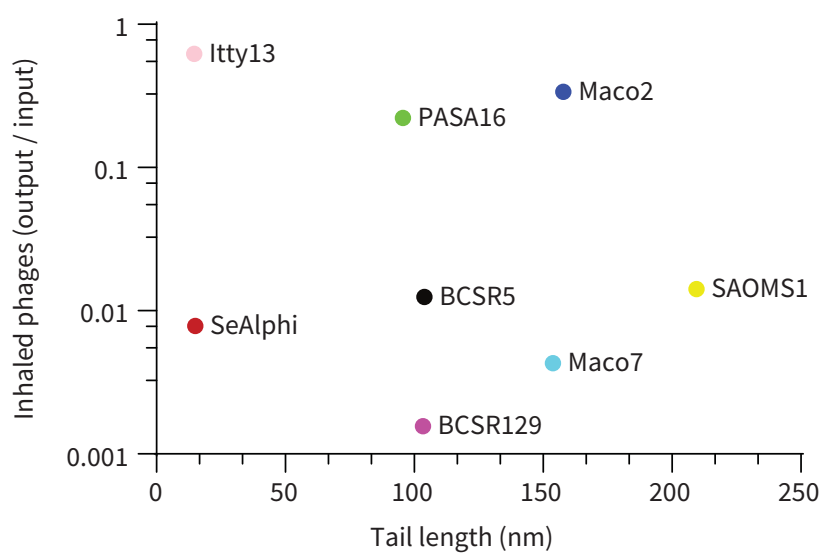

c)

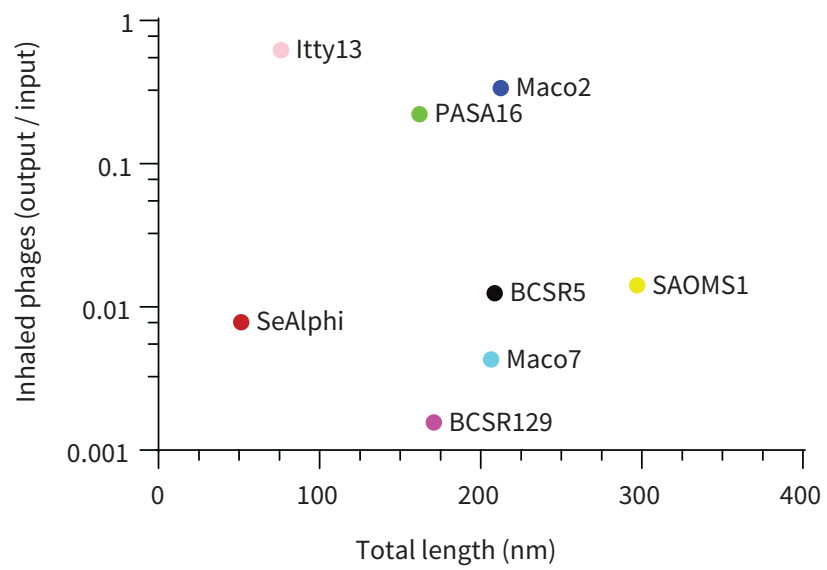

e)

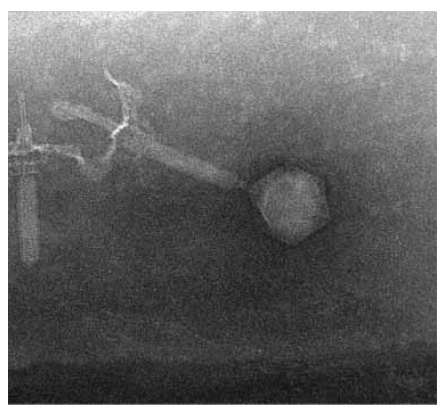

BCSR5

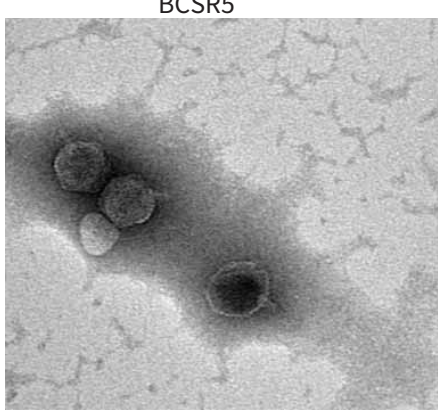

Itty13

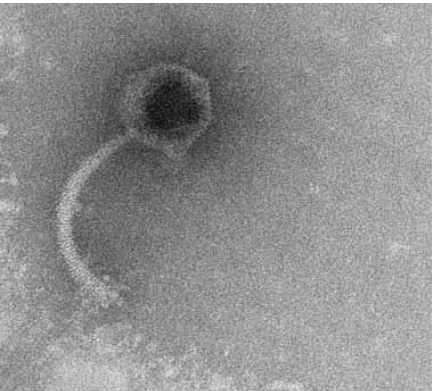

SAOMS1

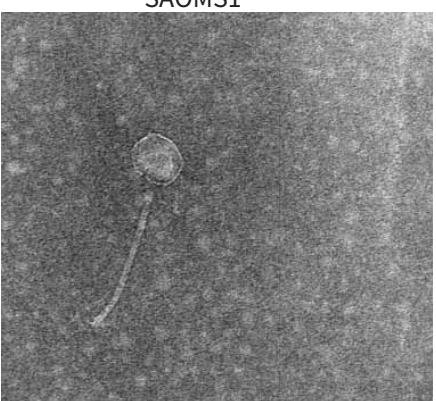

$\mathrm{MACO} 2$ b)

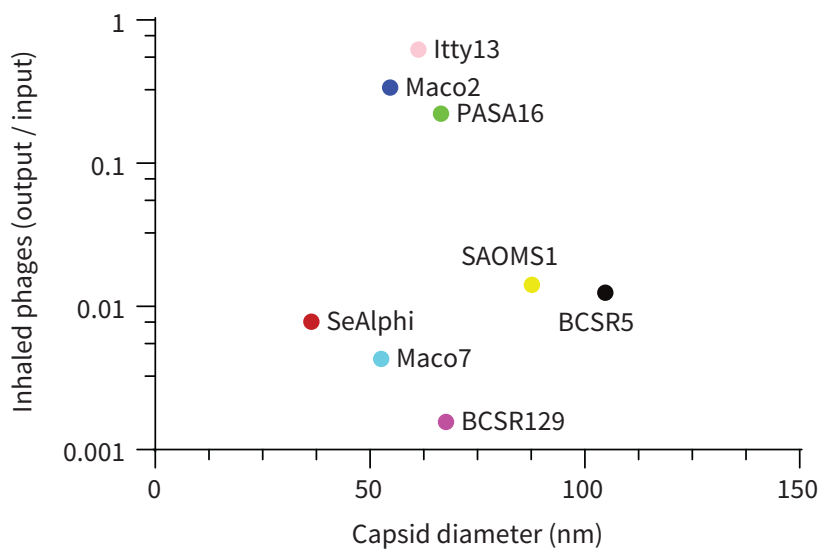

d)

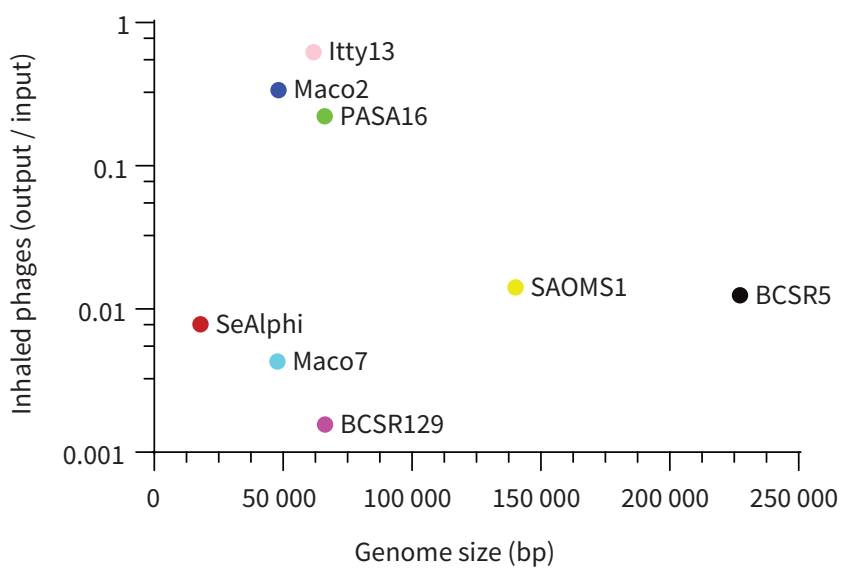

BCSR129

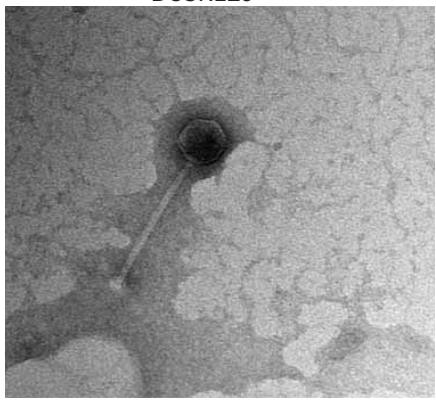

MACO7

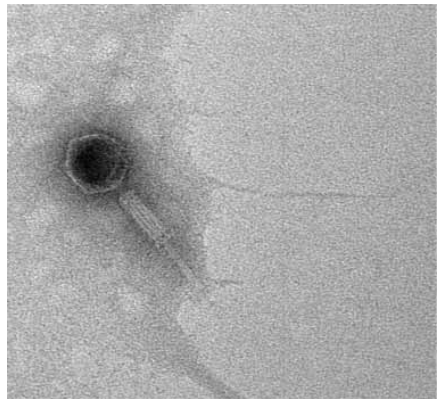

PASA16

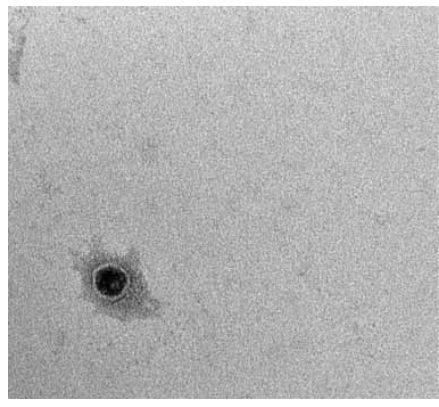

SeAlphi

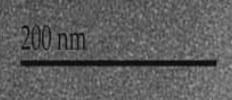


FIGURE 3 Phage characterisation and its effect on inhalation fitness. Correlation plots representing phage inhalation efficacy, defined as the proportion between viable phages reaching the tracheal level and the corrected initial phage loads, according to a) tail length, b) capsid diameter, c) total phage length and d) phage genome size. e) Transmission electron microscopy images of the phages used in the study. Scale bar: $200 \mathrm{~nm}$.

Furthermore, even though many phages from diverse families were included in the study, a more comprehensive evaluation could potentially shed more light on specific characteristics associated with titre decrease during inhalation. Finally, we have used only one nebuliser type, while other nebulisers may behave differently [15]. However, this only reinforces our main conclusion that prior testing of the phage and the inhalation device in a tailored model are essential for proper treatment design.

In conclusion, here we have shown that phages may act in a non-predictive way in terms of lung delivery following inhalation. Thus, analogously to the concept of phage matching based on accurate laboratory testing of phages against the bacterial target [12], in vitro phage delivery assays should also be performed in a personalised manner, potentially by patient-specific airways models in the future. In this regard, proper phage inhalation requires selecting the most suitable phage and the most appropriate inhalation device by empirical in vitro assessments.

Acknowledgements: The authors thank Yossi Aldar for technical assistance and Ori Inbar from the Cystic Fibrosis Foundation in Israel for insightful ideas.

Provenance: Submitted article, peer reviewed.

Conflict of interest: None declared.

Support statement: This study was supported by United States-Israel Binational Science Foundation grant \#2017123, Israel Science Foundation IPMP grant \#ISF_1349/20, Rosetrees Trust grant A2232 and the Milgrom Family Support Program. Funding information for this article has been deposited with the Crossref Funder Registry.

References

1 Altamirano FLG, Barr JJ. Phage therapy in the postantibiotic era. Clin Microbiol Rev 2019; 32: e00066-18.

2 Aslam S, Lampley E, Wooten D, et al. Lessons learned from the first 10 consecutive cases of intravenous bacteriophage therapy to treat multidrug-resistant bacterial infections at a single center in the United States. Open Forum Infect Dis 2020; 7: ofaa389.

3 Djebara S, Maussen C, De Vos D, et al. Processing phage therapy requests in a Brussels military hospital: lessons identified. Viruses 2019; 11: 265.

4 Fabijan AP, Lin RCY, Ho J, et al. Safety of bacteriophage therapy in severe Staphylococcus aureus infection. Nat Microbiol 2020; 5: 465-472.

5 Aslam S, Courtwright AM, Koval C, et al. Early clinical experience of bacteriophage therapy in 3 lung transplant recipients. Am J Transplant Wiley Online Library 2019; 19: 2631-2639.

6 Guillon A, Pardessus J, L'Hostis G, et al. Inhaled bacteriophage therapy in a porcine model of pneumonia caused by Pseudomonas aeruginosa during mechanical ventilation. Br J Pharmacol 2021; 178: 3829-3842.

7 Prazak J, Iten M, Cameron DR, et al. Bacteriophages improve outcomes in experimental Staphylococcus aureus ventilator-associated pneumonia. Am J Respir Crit Care Med 2019; 200: 1126-1133.

8 Hoyle N, Zhvaniya P, Balarjishvili N, et al. Phage therapy against Achromobacter xylosoxidans lung infection in a patient with cystic fibrosis: a case report. Res Microbiol Elsevier 2018; 169: 540-542.

9 Law N, Logan C, Yung G, et al. Successful adjunctive use of bacteriophage therapy for treatment of multidrug-resistant Pseudomonas aeruginosa infection in a cystic fibrosis patient. Infection 2019; 47: 665-668.

10 Lebeaux D, Merabishvili M, Caudron E, et al. A case of phage therapy against pandrug-resistant Achromobacter xylosoxidans in a 12-year-old lung-transplanted cystic fibrosis patient. Viruses 2021; 13: 60.

11 Koff JL, Chan BK, Stanley GL, et al. Clinical use of inhaled bacteriophages to treat multi-drug resistant Pseudomonas aeruginosa. Am J Respir Crit Care Med 2019; 199: A7381.

12 Gelman D, Yerushalmy O, Ben-Porat S, et al. Clinical phage microbiology: a suggested framework and recommendations for the in-vitro matching steps of phage therapy. bioRxiv 2021; preprint [https://doi.org/10. 1101/2021.02.23.432393].

13 Niederman MS. Adjunctive nebulized antibiotics: what is their place in ICU infections? Front Med 2019; 6: 99.

14 Sahota JS, Smith CM, Radhakrishnan P, et al. Bacteriophage delivery by nebulization and efficacy against phenotypically diverse Pseudomonas aeruginosa from cystic fibrosis patients. J Aerosol Med Pulm Drug Deliv 2015; 28: 353-360. 
Carrigy NB, Chang RY, Leung SSY, et al. Anti-tuberculosis bacteriophage D29 delivery with a vibrating mesh nebulizer, jet nebulizer, and soft mist inhaler. Pharm Res 2017; 34: 2084-2096.

16 Amirav I, Borojeni AAT, Halamish A, et al. Nasal versus oral aerosol delivery to the 'lungs' in infants and toddlers. Pediatr Pulmonol 2015; 50: 276-283.

17 Amirav I, Halamish A, Gorenberg M, et al. More realistic face model surface improves relevance of pediatric in-vitro aerosol studies. PLOS ONE 2015; 10: e0128538.

18 Yerushalmy O, Khalifa L, Gold N, et al. The Israeli Phage Bank (IPB). Antibiotics 2020; 9: 269.

19 Khalifa L, Brosh Y, Gelman D, et al. Targeting Enterococcus faecalis biofilms with phage therapy. Appl Environ Microbiol 2015; 81: 2696-2705.

20 Gelman D, Beyth S, Lerer V, et al. Combined bacteriophages and antibiotics as an efficient therapy against VRE Enterococcus faecalis in a mouse model. Res Microbiol 2018; 169: 531-539.

21 Wallin M, Tang P, Chang RYK, et al. Aerosol drug delivery to the lungs during nasal high flow therapy: an in vitro study. BMC Pulm Med 2019; 19: 1-11.

22 Astudillo A, Leung SSY, Kutter E, et al. Nebulization effects on structural stability of bacteriophage PEV 44. Eur J Pharm Biopharm 2018; 125: 124-130.

23 Leung SSY, Carrigy NB, Vehring R, et al. Jet nebulization of bacteriophages with different tail morphologies: structural effects. Int J Pharm 2019; 554: 322-326.

24 Niederman MS, Alder J, Bassetti M, et al. Inhaled amikacin adjunctive to intravenous standard-of-care antibiotics in mechanically ventilated patients with Gram-negative pneumonia (INHALE): a double-blind, randomised, placebo-controlled, phase 3, superiority trial. Lancet Infect Dis 2020; 20: 330-340.

25 Kollef $\mathrm{MH}$, Ricard J-D, Roux D, et al. A randomized trial of the amikacin fosfomycin inhalation system for the adjunctive therapy of Gram-negative ventilator-associated pneumonia: IASIS Trial. Chest 2017; 151: 1239-1246.

26 Lin Y, Quan D, Chang RYK, et al. Synergistic activity of phage PEV20-ciprofloxacin combination powder formulation: a proof-of-principle study in a P. aeruginosa lung infection model. Eur J Pharm Biopharm 2021; 158: $166-171$. 